\title{
Thermal, quantum anti-bunching and lasing thresholds from single emitters to macroscopic devices
}

\author{
Mark Anthony Carroll, ${ }^{1}$ Giampaolo D'Alessandro, ${ }^{2}$ Gian Luca Lippi,${ }^{3}$ Gian-Luca Oppo, ${ }^{1}$ and Francesco Papoff ${ }^{1, *}$ \\ ${ }^{1}$ Department of Physics, University of Strathclyde, 107 Rottenrow, Glasgow G4 0NG, UK. \\ ${ }^{2}$ School of Mathematics, University of Southampton, Southampton SO17 1BJ, United Kingdom \\ ${ }^{3}$ Université Côte d'Azur, Institut de Physique de Nice, \\ UMR 7710 CNRS, 1361 Route des Lucioles, 06560 Valbonne, France
}

(Dated: December 11, 2020)

\begin{abstract}
Starting from a fully quantized Hamiltonian for an ensemble of identical emitters coupled to the modes of an optical cavity, we determine analytically regimes of thermal, collective anti-bunching and laser emission that depend explicitly on the number of emitters. The lasing regime is reached for a number of emitters above a critical number - which depends on the light-matter coupling, detuning and the dissipation rates - via a universal transition from thermal emission to collective anti-bunching to lasing as the pump increases. Cases where the second order intensity correlation fails to predict laser action are also presented.
\end{abstract}

Optical cavities containing emitters with discrete energy levels such as atoms, ions or quantum dots, have proved extremely effective both as a tool to investigate fundamental properties of light-matter interaction and as a way to produce light with engineered statistical properties. Historically, the earliest optical cavities contained very large numbers of emitters to overcome losses and occupied macroscopic volumes. Significant improvements in cavity quality have led in recent years to micro and nano cavities [1] that provide detectable fields even with very few emitters and promise commercial applications with uses ranging from components on integrated circuits to medicine [2]. Their small size comes with the benefit of lower energy consumption and increased energy efficiency, making them attractive for extreme miniaturization. Although the basic quantum interaction process between the cavity modes and the emitters is the same for all cavities, different approximations of the expectation values of light-matter interaction and photon number (correspondiong to the classical intensity) lead to two classes of models: one for micro and nano systems, the other for macroscopic systems. In the latter, e.g. the Maxwell-Bloch semi-classical models for macroscopic systems [3], the only expectation values considered are those of the emitters' raising and lowering operators, representing excitation and de-excitation of an electron, and of the cavity mode creation and destruction operators, representing emission and absorption of a photon. The expectation values of the destruction and creation operators correspond to the complex amplitude of the classical coherent field and its complex conjugate. Similarly, the expectation values of raising and lowering operators correspond to the amplitude of the medium polarization and its complex conjugate. Macroscopic models neglect correlations among these operators, both in the intensity and in the photon-matter coupling, also called the photon-assisted polarization. This approximation al-

\footnotetext{
*f.papoff@strath.ac.uk
}

lows one to predict the threshold for laser emission, but is not suitable for the analysis of non-lasing emission because photon-assisted polarization is essential to model correctly spontaneous emission [4]. Quantum models for micro and nano lasers take the exact opposite approach: they consider only the correlations and neglect the expectation values [4-7] corresponding to the amplitudes of the classical coherent field and polarization. With this approximation non-lasing emission can be modelled, but it is not possible to identify the onset of lasing. The same problem also affects rate equation models of micro- and nanolasers that add average spontaneous emission to coherent emission [8-10]. A key parameter related to the size of the system is the spontaneous emission factor, $\beta$, defined as the ratio of the spontaneous emission rate into the lasing mode to the total spontaneous emission. $\beta^{-1}$, proportional to the number of electromagnetic modes in the cavity volume, thus characterises the system size. In macroscopic systems $\beta \ll 1$, while $\beta=1$ is the nanoscale limit, in which only the lasing mode remains accessible to spontaneous and stimulated emission. For this value of $\beta$ the output power linearly follows the input power, and for this reason this laser is considered "thresholdless" $[8,11]$. This regime poses questions on how to define the laser threshold [9] and identify coherent emission [12], which we address unambiguously in this paper.

Starting from a fully quantized Jaynes-Cummings Hamiltonian in the Heisenberg picture, see Supplementary Material (SM) Eq.(1), we derive a model for the emission of any number of identical two-level emitters coupled to one mode of the cavity $[13,14]$. However, we do not approximate the expectation values of intensity and light-matter interaction, including the variables of both macroscopic and nano lasers, and apply dynamical system methods defined for systems of any dimension [15] to identify the laser threshold. Analytical solutions seamlessly connect single emitter devices with devices containing millions of emitters, predicting where the thermal, quantum and coherent emission regimes lie with respect to one another. While the extent and exis- 
tence of these regions in the space of parameters depend on the number of emitters, we find two universal features that are common to all lasing devices. The first is that lasing, when possible, is reached via a universal sequence of transitions as the pump is increased. The emission of the non-lasing state evolves continuously from thermal (with second order intensity correlation $1<g^{(2)}(0) \leq 2$ ) to anti-bunching (with $0 \leq g^{(2)}(0)<1$ ) until the laser threshold is crossed and the non-lasing state becomes unstable. A coherent laser field, due to a lasing collective state, appears at this threshold and its amplitude increases as a function of the pump. This is the same instability predicted for macroscopic laser by MaxwellBloch models. However, in these models the total field before the instability is zero, while in our theory before the threshold only the coherent field is zero, while the incoherent field is non zero. The second universal feature of lasers is that the emerging coherent field has a well defined frequency: our model shows that neither the number of emitters nor the effective cavity volume ( $\beta$ factor) influence the frequency value, which remains a general feature of the cavity-emitter interaction. We give examples where the measurement of $g^{(2)}(0)$ cannot identify laser emission.

We consider identical emitters with two energy levels and one electron, and assume that all transitions conserve the electron spin. This model applies to atoms and ions with suitable energy level structure, and also to shallow quantum dots (possessing two localized levels) at temperatures low enough to neglect Coulomb and phonon interactions. Assuming that detuning and coupling coefficients with the mode are identical $[7,16]$ is justified by numerical simulations for emitters with $10 \%$ random variations of detuning and coupling coefficients. In these simulations all emitters' variables - started from random initial conditions - after a transient converge to common values that match extremely well those obtained using the same parameters and expectation values for all emitters, see SM Fig. 2. Since current technology enables very accurate positioning [31], the range of fluctuations that we are considering is quite realistic and covers estimates which can be expected from QD positions inside typical cavity realizations and other experimental fluctuations.

In the following $c^{\dagger}, v^{\dagger}, b^{\dagger}(c, v, b)$ are operators that create (annihilate), respectively, an electron in the upper energy level (conduction level in quantum dot terminology), in the lower energy level (valence level), and a photon in the laser mode. We take the expectation values of the Heisenberg equations for each operator in the Hamiltonian, truncating the resulting infinite hierarchy of coupled differential equations by keeping only correlation functions that appear in the cluster expansion of the Hamiltonian $[4,13]$. The variables of the model are the expectation values of carrier population, $\left\langle c^{\dagger} c\right\rangle$, mode destruction operator, $\langle b\rangle$, and emitter lowering operator, $\left\langle v^{\dagger} c\right\rangle$, and the intensity and photon-polarization correlations, $\delta\left\langle b^{\dagger} b\right\rangle$ and $\delta\left\langle b c^{\dagger} v\right\rangle$. These correlations appear through the cluster expansions $\left\langle b c^{\dagger} v\right\rangle=\delta\left\langle b c^{\dagger} v\right\rangle+\langle b\rangle\left\langle c^{\dagger} v\right\rangle$ and $\left\langle b^{\dagger} b\right\rangle=\delta\left\langle b^{\dagger} b\right\rangle+\left\langle b^{\dagger}\right\rangle\langle b\rangle$ of terms in the Hamiltonian. Note that the equations for $\left\langle b^{\dagger}\right\rangle,\left\langle c^{\dagger} v\right\rangle,\left\langle b^{\dagger} v^{\dagger} c\right\rangle$ are the complex conjugated of the equation for $\langle b\rangle,\left\langle v^{\dagger} c\right\rangle,\left\langle b c^{\dagger} v\right\rangle$, so they are not explicitly included in the model. The model equations are

$$
\begin{aligned}
d_{t}\left\langle c^{\dagger} c\right\rangle= & -\left(\gamma_{n l}+\gamma_{n r}\right)\left\langle c^{\dagger} c\right\rangle+r\left(1-\left\langle c^{\dagger} c\right\rangle\right) \\
& -2 \mathfrak{R e}\left[g\left(\delta\left\langle b c^{\dagger} v\right\rangle+\langle b\rangle\left\langle c^{\dagger} v\right\rangle\right)\right], \\
d_{t} \delta\left\langle b c^{\dagger} v\right\rangle= & -\left(\gamma_{c}+\gamma+i \Delta \nu\right) \delta\left\langle b c^{\dagger} v\right\rangle+g^{*}\left[\left\langle c^{\dagger} c\right\rangle\right. \\
& \left.+\delta\left\langle b^{\dagger} b\right\rangle\left(2\left\langle c^{\dagger} c\right\rangle-1\right)-\left|\left\langle v^{\dagger} c\right\rangle\right|^{2}\right], \\
d_{t} \delta\left\langle b^{\dagger} b\right\rangle= & -2 \gamma_{c} \delta\left\langle b^{\dagger} b\right\rangle+2 N \Re \mathfrak{R e}\left(g \delta\left\langle b c^{\dagger} v\right\rangle\right), \\
d_{t}\langle b\rangle= & -\left(\gamma_{c}+i \nu\right)\langle b\rangle+g^{*} N\left\langle v^{\dagger} c\right\rangle, \\
d_{t}\left\langle v^{\dagger} c\right\rangle= & -\left(\gamma+i \nu_{\varepsilon}\right)\left\langle v^{\dagger} c\right\rangle+g\left[\langle b\rangle\left(2\left\langle c^{\dagger} c\right\rangle-1\right)\right],
\end{aligned}
$$

where $r$ is the pump rate per emitter (the one for the whole system is $N r$ ), with non resonant pump photons absorption and carrier transport effects approximated via an injection rate [7]. $\quad \nu$ is the frequency of the resonant mode and $\nu_{\varepsilon}$ is the resonant frequency of emitters, $\Delta \nu=\nu_{\varepsilon}-\nu$ is the detuning between the field and emitter, $g$ the light-matter coupling strength and $N$ the number of emitters. The decay rates for the laser mode, $\gamma_{c}$, the population, $\gamma_{n r}$, and polarisation, $\gamma$, introduce dissipation. The dissipative part of these equations can be obtained by considering Lindblad terms describing the coupling to a Markovian bath $[17,18] . \quad \gamma_{n r}$ is due to non-radiative transitions, while the additional decay rate for the population, $\gamma_{n l}$, results from the adiabatic elimination of rapidly decaying non lasing modes [19]. The spontaneous emission factor, $\beta$, is related to the cavity losses by $\beta=\frac{\gamma_{l}}{\gamma_{n l}+\gamma_{l}}$, where $\gamma_{l}$ is the rate of spontaneous emission into the lasing mode. The expectation value of lower level population, $\left\langle v^{\dagger} v\right\rangle$ has been eliminated using $\left\langle c^{\dagger} c\right\rangle+\left\langle v^{\dagger} v\right\rangle=1$. The variables $\langle b\rangle$ and $\left\langle v^{\dagger} c\right\rangle$ correspond to the amplitudes of the coherent field and the medium polarization of semi-classical models of macroscopic lasers and were previously neglected in nanolasers. The imaginary coefficients in their the equations show that they have fast oscillations with frequency of the order of that of the cavity mode. Hence, we call them "fast" and the remaining variables "slow".

The non-lasing stationary state is found by setting to zero the fast variables and the time derivatives in Eqs.(1$3)$. This state exists for all values of the control parameters and its emission evolves continuously from thermal to anti-bunching. The boundary between these two regimes is identified by the curve $g^{(2)}(0)=1$. We find a very good analytical approximation of the $g^{(2)}(0)=1$ curve assuming that the correlations used here are independent of higher order correlations [6], so that $g^{(2)}(0) \sim 2+A / B$, see SM Eqs.(12-14). We determine the stability of the non-lasing state by deriving the equations that govern the evolution of small perturbations in the linear regime. We find that the perturbations of the fast variables are decou- 

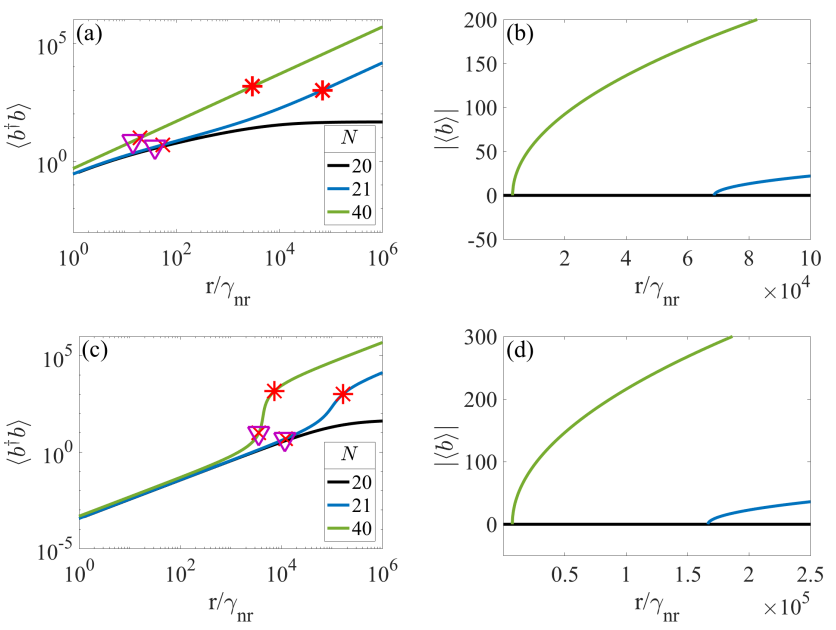

FIG. 1. I/O curves: Intensity, $\left\langle b^{\dagger} b\right\rangle=\delta\left\langle b^{\dagger} b\right\rangle+\left\langle b^{\dagger}\right\rangle\langle b\rangle$, and coherent field amplitude for different $N$ above and below $N_{c}$ versus pump for $\beta=1\left(\gamma_{n l}=0\right)$, (a) and (b); and $\beta=7 \times 10^{-4}$ $\left(\gamma_{n l}=1.4 \times 10^{12} s^{-1}\right.$ and $\left.\gamma_{l}=9.68 \times 10^{8} s^{-1}\right),(\mathrm{c})$ and $(\mathrm{d})$. Laser and anti-bunching thresholds are marked by red stars and crosses, respectively. The purple triangles mark the antibunching threshold where we include higher order correlations (this shows our approximation to be valid). Note that a laser threshold is found even for a "thresholdless" device (green line in (a)). Parameters: $\gamma=10^{13} s^{-1}, \gamma_{c}=10^{10} s^{-1}, \gamma_{n r}=$ $10^{9} \mathrm{~s}^{-1}$, and $g=7 \times 10^{10} \mathrm{~s}^{-1}$. Losses are kept constant in all figures.

pled from the perturbations of the slow variables allowing us to determine analytically the stability of the nonlasing state (see SM Eqs.(22-24)). The non-lasing state is stable for $\left\langle c^{\dagger} c\right\rangle\left\langle\left\langle c^{\dagger} c\right\rangle_{t h}\right.$ and unstable for $\left\langle c^{\dagger} c\right\rangle>\left\langle c^{\dagger} c\right\rangle_{t h}$ where

$$
\left\langle c^{\dagger} c\right\rangle_{t h}=\frac{1}{2}+\frac{\gamma_{c} \gamma}{2 N|g|^{2}}\left[1+\left(\frac{\Delta \nu}{\gamma_{c}+\gamma}\right)^{2}\right]
$$

is the laser threshold. Because $\left\langle c^{\dagger} c\right\rangle<1$, lasing can only happen when the number of emitters satisfies the condition

$$
\frac{\gamma_{c} \gamma}{|g|^{2}}\left[1+\left(\frac{\Delta \nu}{\gamma_{c}+\gamma}\right)^{2}\right] \leq N
$$

The laser frequency is determined using trial solutions $\langle b\rangle,\left\langle v^{\dagger} c\right\rangle \propto e^{-i \Omega t}$ in Eqs. $(4,5)$ and is

$$
\Omega=\nu+\frac{\gamma_{c} \Delta \nu}{\gamma_{c}+\gamma}
$$

A few points are worth highlighting. First, the threshold can be calculated for any values of the decay rate $\gamma_{n l}$, including the so called "thresholdless" case $\gamma_{n l}=0$. Second, neither $\left\langle c^{\dagger} c\right\rangle_{t h}$ nor the critical number of emitters necessary to lase depend on $\beta$. However, the value of the pump per emitter required to reach $\left\langle c^{\dagger} c\right\rangle_{t h}$ depends on $\beta$ and decreases as $\beta$ increases, see SM Eq.(20) for the analytic expression for $r$ at laser threshold. Third, the laser
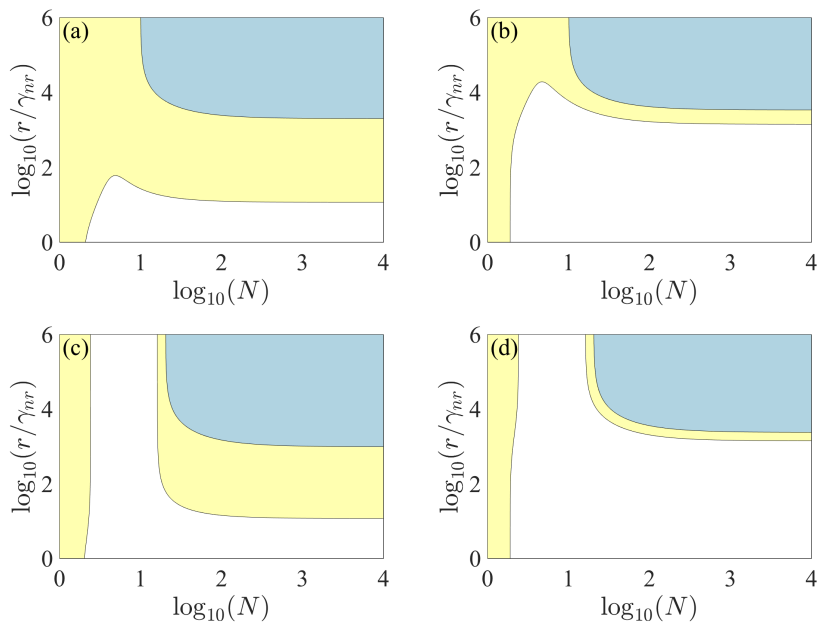

FIG. 2. Phase Diagrams: thermal, anti-bunching and lasing regimes correspond to the white, yellow and blue regions, respectively. Where $\beta=1$ and $g=10^{11} s^{-1}$ in (a); $\beta=7 \times 10^{-4}$ and $g=10^{11} s^{-1}$ in (b); $\beta=1$ and $g=7 \times 10^{10} s^{-1}$ in (c); $\beta=7 \times 10^{-4}$ and $g=7 \times 10^{10} s^{-1}$ in (d). For large $g$ the thermal region that extends to large pump values vanishes, and for a lasing a device with $\beta=1$ the anti-bunching regime exists over greater values of the pump.

frequency $\Omega$ is independent of the number of emitters, $N$.

In Fig. 1a and Fig. 1b the intensity and coherent field versus pump for a $\beta=1$ device are shown. The different number of emitters correspond to values above (blue and olive curves) and below (black curves) the critical number, $N_{c}$, required for a laser using Eq.(7). Below $N_{c}$, the minimum integer that satisfies Eq.(7), the coherent field amplitude is always zero and the intensity saturates at high pump. Above $N_{c},\langle b\rangle \neq 0$ emerges through a pitchfork bifurcation. The emergence of a pitchfork bifurcation also coincides with the growth of the intensity and a clear qualitative difference is apparent between the curves corresponding to lasing and non lasing devices. The I/O curve for 40 quantum dots (Fig. 1a) illustrates the impossible task of determining the laser threshold for a $\beta=1$ device, identifiable only through the fast variables. Comparison of these thresholds with standard estimates is given in SM Fig. 4. Notice that all graphs plot pump for a single quantum dot. Thus, comparison between devices with different $N$ requires multiplication of each horizontal scale by $N$. Variations in the I/O similar to those shown in Fig. 1a for $N=21$ and $N=40$ can be obtained with the same value of $\mathrm{N}$ and changing the detuning. This is observed in experiments where detuning decreases the effective number of quantum dots interacting with the field. See SM Fig. 6 for the effect of detuning. Experiments have obtained this kind of I/O response through cavity or thermal tuning [20-22]. While so far explained only through ad-hoc calculations, here the continuous transformation with change in characteristic I/O shape emerges thanks to self-consistent model- 

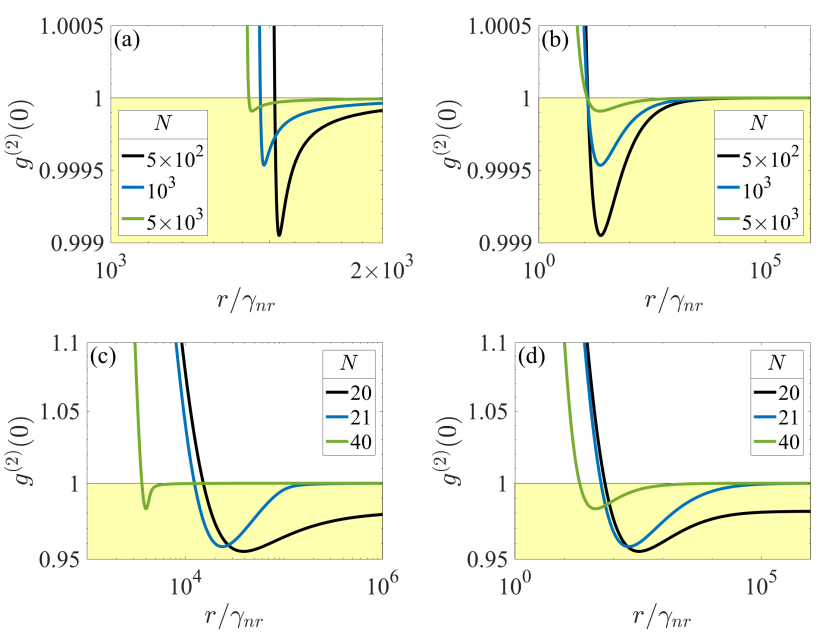

FIG. 3. $g^{(2)}(0)$ versus pump for different $N$ and $\beta$ where the coloured regions correspond to the same in Fig. 2. In (a) and (c) $\beta=7 \times 10^{-4}$; and in (b) and (d) $\beta=1$. Note the large range of pump values in (a) and (b) where $g^{(2)}(0)$ is smaller, but very close to 1 , making $g^{(2)}(0)$ difficult to use as an experimental indicator of lasing. All curves in (a) converge to the same value for larger pump values not shown. All curves are obtained for $g=7 \times 10^{10} s^{-1}$ thus lasing occurs for $N=21$.

ing.

Fig. 1c and Fig. 1d show the intensity and coherent field for a device where $\beta \ll 1$. One clear difference is that the intensity profile is no longer linear and there is the characteristic s-shaped I/O curve. Notable is the appearance of the bifurcation at the knee of the upper branch of the I/O curve, rather than at the inflection point (as from [9]). This points to a substantial contribution from the incoherent emission to the intensity growth before the bifurcation and highlights the difficulty intrinsic in determining threshold through the I/O curve.

Fig. 2 shows the analytic solutions of $g^{(2)}(0)=1$ and the laser threshold as functions of the pump and $N$ for different values of $g$ and $\beta$. For a device capable of lasing there is a well defined path of emission as the pump increases (vertical cut in the graph): from thermal to anti-bunching; then from anti-bunching to lasing. This is independent of the value of $\beta$. Fig. 2a and Fig. $2 \mathrm{c}$ show an anti-bunching regime that exists for a large region of the pump and extends from nano- to macroscopic lasers with $\beta=1$. Anti-bunching has been observed in an experiment with a high cavity $\mathrm{Q}$ micropillar containing $N \approx 15$ pumped quantum dots [23] and in numerical simulations of quantum dot nanocavities [6],[24]. The observation is consistent with our prediction (Fig. 3), which shows stronger anti-bunching close to $N_{c}$; an increase in $N$ reduces the range and amplitude, thus making an experi- mental observation much more difficult (no anti-bunching was in fact observed upon doubling of the quantum dot number [23]). Fig. 3a shows the three regimes for lasers with $\beta=7 \times 10^{-4}$. Although anti-bunching is present in macroscopic lasers, $g^{(2)}(0)$ is so close to unity that it becomes very difficult to distinguish between a laser and a device emitting anti-bunched light, thus stressing that $g^{(2)}(0)$ is not a sufficient indicator of laser action. We show in Fig. 3 of the SM a comparison of Eqs. (1-5) with the master equation given in [9] and a cQED model [7].

In conclusion, we have derived a model that includes coherent and incoherent emission and derived analytically thresholds that separate thermal, anti-bunching and lasing emission regimes from single emitter to macroscopic devices. We predict the qualitative differences between the I/O curves of the photon number above and below threshold and find analytically the number of intracavity emitters necessary for laser action to occur. We identify a universal route leading from thermal to coherent emission, through a collective anti-bunching regime that always precedes lasing. The coherent laser field always emerges from a bifurcation with a well defined threshold and has a frequency independent from the number of emitters. Interferometric measurements of the coherence time $[25,26]\left(g^{(1)}(\tau)\right)$ - equivalent to detecting the existence of a well-defined frequency at the bifurcation - can in principle be used to unambiguously identify lasing in all devices. This also holds for $\beta=1$ (or close to this boundary), where the $\mathrm{I} / \mathrm{O}$ characteristics cannot carry useful information and measurements of $g^{(2)}(0)$ are poor indicators of lasing, leading to wrong conclusions on the existence or position of the laser threshold. The accurate characterization of the coherence properties of nanolasers holds promise for quantitative predictions to be used in a variety of applications, ranging from telecommunications and optical chips [27], to the spectroscopic use of nanosources [28], nanosensing [29] and biophysical applications [30]. These applications can greatly benefit from a detailed understanding and predictive power of designs where control in the number of quantum dots (now technologically feasible $[31,32])$ coupled to effective coupling allow for tailored emission properties. In addition, at the micro and nano scale this new theory can be used to investigate the interaction of emitters with nanostructures and arrays of particles [33-37] and obtain predictions for new effects emerging from quantum interactions.

The Strathclyde and UCA groups are grateful to the the CNRS and its Laboratoire International Associé (LIA) "Solace" for support. The PhD of Mark Anthony Carroll is supported by the University of Strathclyde. GD and FP wish to thank the Mathematisches Forschungsinstitut Oberwolfach for support. GLL is grateful to L. Chusseau for discussions. 
[1] M.T. Hill and M.C. Gather. Advances in small lasers. Nature Photon., 8:908-918, 2014.

[2] R.M. Ma and R.F. Oulton. Applications of nanolasers. Nature Nanotechnol., 14:12-22, 2019.

[3] L.M. Narducci and N.B. Abraham. Laser Physics and Laser Instabilities. (World Scientific, 1988.

[4] Mackillo Kira and Stephan W Koch. Semiconductor quantum optics. Cambridge University Press, 2011.

[5] C. Gies, J. Wiersig, M. Lorke, and F. Jahnke. Semiconductor model for quantum-dot-based microcavity lasers. Phys. Rev. A, 75:013803, 2007.

[6] W.W Chow, F. Jahnke, and C. Gies. Emission properties of nanolasers during the transition to lasing. Light: Science \& Applications, 3:e201, 2014.

[7] S. Kreinberg, W.W. Chow, J. Wolters, C. Schneider, C. Gies, F. Jahnke, S. Höfling, M. Kamp, and S. Reitzenstein. Emission from quantum-dot high- $\beta$ microcavities: transition from spontaneous emission to lasing and the effects of superradiant emitter coupling. Light: Science E Applications, 6:e17030, 2017.

[8] H. Yokoyama and S. D. Brorson. Rate equation analysis of microcavity laser. J. Appl. Phys., 66:4801-4805, 1989.

[9] Perry R Rice and HJ Carmichael. Photon statistics of a cavity-qed laser: A comment on the laser-phasetransition analogy. Physical Review A, 50(5):4318, 1994.

[10] J. Mork and G.L. Lippi. Rate equation description of quantum noise in nanolasers with few emitters. Applied Physics Letters, 112(14):141103, 2018.

[11] C.Z. Ning. What is laser threshold? J. Sel. Topics Quantum Electron., 19:1500304, 2013.

[12] I.D.W. Samuel and G.A. Turnbull E.B. Namdas. How to recognize lasing. Nature Photonics, 3:546-549, 2009.

[13] J. Fricke. Transport Equations Including Many-Particle Correlations for an Arbitrary Quantum System: A General Formalism. Annals of physics, 252:479-498, 1996.

[14] T. Feldtmann, L. Schneebeli, M. Kira, and S. W. Koch. Quantum theory of light emission from a semiconductor quantum dot. Phys. Rev. B, 73:155319, 2006.

[15] H. Solari, M. Natiello, and G. Mindlin. Nonlinear Dynamics: A Two-way Trip from Physics to Math. CRC Press, 1996.

[16] G. Moody, and I. Sagnes M. Segnon, R. BraiveA, A. Beveratos, I. Robert-Philip, N. Belabas, F. Janke, K.L. Silverman, R.P. Mirin, M.J. Stevens, and C. Gies. Delayed formation of coherence in the emission dynamics of highQ nanolasers. Optica, 5:395-401, 2018.

[17] M. Florian, C. Gies, F. Jahnke, H.A.M. Leymann, and J. Wiersig. Equation-of-motion technique for finite-size quantum-dot systems: Cluster expansion method. Phys. Rev. B, 87:165306, 2013.

[18] H.A.M. Leymann, A. Foerster, and J. Wiersig. Expectation value based equation-of-motion approach for open quantum systems: A general formalism. Phys. Rev. B, 89:085308, 2014.

[19] W.W Chow and F. Jahnke. On the physics of semiconductor quantum dots for applications in lasers and quantum optics. Prog. Quantum. Electron., 37:109-184, 2014.

[20] M. Takiguchi et al. Systematic study of thresholdless oscillation in high- $\beta$ buried multiple-quantum-well pho- tonic crystal nanocavity lasers. Opt. Express, 24:34413450, 2016.

[21] Y. Ota, M. Kakuda, K. Watanabe, S.Iwamoto, and Y. Arakawa. Thresholdless quantum dot nanolaser. Opt. Express, 25:19981-19994, 2017.

[22] S.T. Jagsch et al. A quantum optical study of thresholdless lasing features in high- $\beta$ nitride nanobeam cavities. Nature Commun., 9:564, 2018.

[23] J. Wiersig et al. Direct observation of correlations between individual photon emission events of a microcavity laser. Nature, 460:245-250, 2009.

[24] C. Gies, F. Jahnke, and W.W. Chow. Photon antibunching from few quantum dots in a cavity. Phys. Rev. A, 91:061804(R), 2015.

[25] A. Lebreton, I. Abram, R. Braive, I. Sagnes, I. RobertPhilip, and A. Beveratos. Unequivocal Differentiation of Coherent and Chaotic Light through Interferometric Photon Correlation Measurements. Phys. Rev. Lett., 110:163603, 2013.

[26] S.M. Ulrich, C. Gies, S. Ates, J. Wiersig, S. Reitzenstein, C. Hofmann, A. Löffler, A. Forchel, F. Jahnke, and P. Michler. Photon statistics of semiconductor microcavity lasers. Phys. Rev. Lett., 98:043906, 2007.

[27] D.A.B. Miller. Attojoule optoelectronics for low-energy information processing and communications. J. Lightwave Technol., 35:346-396, 2017.

[28] P. Melentiev et al. Plasmonic nanolaser for intracavityspectroscopy and sensorics. Appl. Phys. Lett., 111:213104, 2017.

[29] M.I. Stockman. Nanoplasmonic sensing and detection. Science, 348:287-288, 2015.

[30] A.H. Fikouras, M. Schubert, M. Karl, J.D. Kumar, S.J. Powis, A. Di Falco, and M.C. Gather. Non-obstructive intracellular nanolasers. Nature Commun., 9:4817, 2018.

[31] A. Kaganskiy, S. Kreinberg, X. Porte, and S. Reitzenstein. Micropillar lasers with site-controlled quantum dots as active medium. Optica, 6(4):404-409, 2019.

[32] J. Große, M. von Helversen, A. Koulas-Simos, M. Hermann, and S. Reitzenstein. Development of sitecontrolled quantum dot arrays acting as scalable sources of indistinguishable photons. APL Photonics, 5:096107, 2020.

[33] F. Papoff and B. Hourahine. Geometrical Mie theory for resonances in nanoparticles of any shape. Opt. Express, 19:21432-21444, 2011.

[34] K. Imura, K. Ueno, H. Misawa, H. Okamoto, D. McArthur, B. Hourahine, and F. Papoff. Plasmon modes in single gold nanodiscs. Opt. Express, 22:1218912199, 2014.

[35] D. McArthur, B. Hourahine, and F. Papoff. Enhancing ultraviolet spontaneous emission with a designed quantum vacuum. Opt. Express, 25:12189-12199, 2017.

[36] D. McArthur and F. Papoff. Gap enhanced fluorescence as a road map for the detection of very weakly fluorescent emitters from visible to ultraviolet. Sci. Rep., 7:14191, 2017.

[37] D. McArthur, A.M. Yao, and F. Papoff. Scattering of light with angular momentum from an array of particles. Phys. Rev. Research, 2:013100, 2020. 